\title{
Fathoming the Role of Host Defence Peptides in Periodontal Health and Disease
}

ISSN: 2637-7764

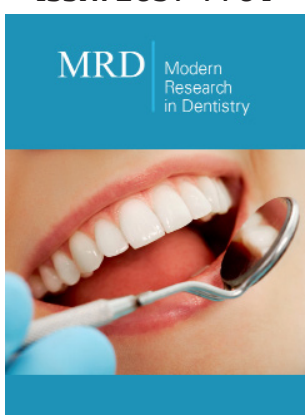

*Corresponding author: CN Guruprasad, Associate professor, Department of Periodontology, GDCRI, Fort, Bangalore-560002, India

Submission: 兆 December 09, 2020

Published: 侮February 15, 2021

Volume 6 - Issue 1

How to cite this article: $C N$ Guruprasad, $M$ Bhavani, S Ramadevi. Fathoming the Role of Host Defence Peptides in Periodontal Health and Disease. Mod Res Dent. 6(1). MRD. 000629. 2021.

DOI: 10.31031/MRD.2021.06.000629

Copyright@ CN Guruprasad, This article is distributed under the terms of the Creative Commons Attribution 4.0 International License, which permits unrestricted use and redistribution provided that the original author and source are credited.

\author{
CN Guruprasad ${ }^{1 *}$, M Bhavani $^{2}$ and S Ramadevi ${ }^{3}$ \\ ${ }^{1}$ Associate professor, Department of Periodontology, GDCRI, Bangalore \\ ${ }^{2}$ Post graduate student, Department of Periodontology, GDCRI, Bangalore \\ ${ }^{3}$ Post graduate student, Department of Orthodontics, The Oxford Dental College and Hospital, \\ Bangalore
}

\begin{abstract}
The periodontal epithelium neighbouring the tooth is precise to form a seal and attachment around the tooth. This function is challenged by development of bacterial biofilm and the host response to biofilm bacteria and their toxins leading to certain vulnerabilities allied with periodontal disease. Oral epithelial cells counters the microbial challenges from dental plaque by the production of host defence peptides (HDP's), chemokines and cytokines that boost inflammation and immune response of periodontal tissues. Host defence peptides are diverse group of biologically active molecules with multidimensional properties. Besides their direct antimicrobial function, they have multiple roles as mediators of inflammation with impact on epithelial and inflammatory cells influencing diverse processes such as cytokine release, cell proliferation, angiogenesis, wound healing, chemotaxis, immune induction, and protease-antiprotease balance. In the oral cavity, the HDPs are produced by the salivary glands and the oral epithelium and they are Defensins and Cathelicidin. This mini review summarizes the current understanding of various oral host defence peptides involved in maintaining balance between periodontal health and disease.
\end{abstract}

Keywords: Host defence peptides; Inflammation; Innate immunity

\section{Introduction}

The warm and moist environment of the oral cavity acts as a shielding interface between the external and internal environment and is the only area of the body in which hard tissues rupture through the epithelial surface. The tooth structure provides a unique niche suitable for number microorganisms to colonize, proliferate and live in harmony as a community ,so called Biofilm [1].The oral epithelium adjacent the tooth is precise to form a seal and attachment around the tooth and acts as physical barricade between the microbial biofilm in the external environment and underlying connective tissue. This exclusive function leads to certain vulnerabilities allied with periodontal disease and imparts unique challenges to the tissue, particularly in view of incessant exposure to the bacterial biofilm. The epithelium combats to microbial oppositions from dental plaque by production of chemokines, cytokines and host defence peptides (HDPs). These HDPs are considered to be paramount for the innate immunity of the host [2].

Host defence peptides, also known as antimicrobial peptides (AMPs), are evolutionarily conserved molecules of the innate immune system. HDPs are always produced as cocktails, and each tissue has its own profile of different HDPs that may vary significantly depending on the actual physiological conditions. More than 106 human HDPs have been isolated to date, of which at least 45 HDPs are present in human saliva and gingival crevicular fluid (GCF) [3]. Most HDPs are cationic molecules with spatially separated hydrophobic and charged regions. This group of proteins and peptides has engendered considerable interest in the past decade as a biological paradigm in innate immunity and as endogenous antibiotics [4].

\section{Antimicrobial action of HDPs}

Many mechanisms of action of HDPs against microorganisms have been reported. However, the following mechanisms are most widely accepted. In barrel-stave model, peptides position themselves for binding on the cell membranes, this leads to peptide aggregation and conversion to a bilayer membrane. So, in this way the hydrophobic peptides align with the lipid 
core and hydrophilic peptides form an access pore in the interior part of membrane. The carpet model is described as a disruption of the membrane by the binding of peptides to the outer surface (phospholipids) of cell membrane and forming a prolonged layer or carpet. In the toroidal model, attached peptides start aggregation and force the lipid monolayer to bend incessantly through the pores. In this way the core is lined by both the inserted peptides and the lipid head groups [5].

HDPs not only lyse the bacteria but also neutralize endotoxins, including lipopolysaccharides (LPS) from gram negative bacteria. The cell lytic action of HDPs is definitely important in the phagosomes of leukocytes and in the core of inflammation, e.g., in the periodontal lesion. However, when it comes to the complex and intricate homeostasis between the microbiome and HDPs in the oral cavity, the neutralizing effects of HDPs on bacterial endotoxins may be more important, as it occurs at lower concentrations of HDPs typically associated with health. Also, the HDPs have several additional features like they are chemotactic for leukocytes or are involved in the regulation of cell proliferation, epithelialization, angiogenesis, wound healing, or adaptive immunity [6].

\section{HDPs in periodontium}

Natural innate environment of the host, the oral epithelium, polymorph nuclear leukocytes (neutrophils) and saliva, all concurrently and solitarily bestow to the biofilm bacteria by production of HDPs. This involves several salivary antimicrobial peptides, the $\beta$-defensins manifested in the epithelium, the $\alpha$-defensins expressed in neutrophils, and the Cathelicidin, LL37 , in both epithelium and neutrophils [7]. The oral epithelium is highly specific, and the AMP expression of anti-microbial proteins is differentially regulated by different periodontal pathogens suggesting that a specific antimicrobial "cocktail" constitutes the physiological response to individual pathogens. The gingival sulcus has a challenging mission-to maintain the epithelial barrier around the tooth, which penetrates the mucosa. To hinder the down-growth of bacteria and to sustain the junctional epithelial barrier there is a high presence of AMPs in the sulcus, due to the high inflammatory activity, even at clinically healthy sites. The high bacterial load in sulcus in itself also induces AMP-expression through toll-like receptor (TLR) and nucleotide oligomerization domain (NOD) signalling, causing a feedback loop [5] (Table 1).

Table 1: Expression sites of Host defence peptides.

\begin{tabular}{|c|c|}
\hline Antimicrobial Peptides & Site of Expression \\
\hline$\alpha$-Defensins (HNP-1) & Neutrophils (azurophilic granules), gingival crevicular fluid \\
\hline$\alpha$-Defensins (HNP-2) & Neutrophils (azurophilic granules), gingival crevicular fluid \\
\hline$\alpha$-Defensins (HNP-3) & Neutrophils (azurophilic granules), gingival crevicular fluid \\
\hline$\alpha$-Defensins (HNP-4) & Neutrophils \\
\hline$\beta$-Defensins (hBD-1) & Guprabasal layer of stratified epithelium and saliva \\
\hline$\beta$-Defensins (hBD-2) & Salivary gland \\
\hline$\beta$-Defensins (hBD-3) & Saliva (parotid and submandibular) \\
\hline Histatin-1 & Saliva (parotid and submandibular) \\
\hline Histatin-3 & Ealiva (parotid and submandibular) \\
\hline Histatin-5 & Neutrophils, inflamed epithelia, submandibular glands and saliva \\
\hline Adrenomedullin & \\
\hline Cathelicidins (LL-37) & \\
\hline
\end{tabular}

\section{Defensins}

Defensins are short, cationic, low molecular weight $(4-5 \mathrm{kDa})$ peptides with 6-8 cysteine residues which form 3-4 intramolecular disulfide bonds. Human defensins are classified as $\alpha, \beta$ and $\theta$ on the basis of their length, location, position of cysteine and folding of peptide chains. These innate defence molecules can kill all kinds of Grampositive and negative bacteria, fungi as well as viruses such as herpes simplex [8].

Alphadefensins are further subclassified into six types, four of the six $\alpha$-defensins, human neutrophil peptide $-1,-2,-3$, and -4 , are synthesized and stored in neutrophil granules. These neutrophils are nearly identical in amino acid sequences, but the N-terminus of hNP-1 ends with alanine (Ala) and aspartate (Asp) for hNP-3.
These changes affect defensin antimicrobial spectrum. In a healthy human, hNP-1 to 3 is most abundantly present in saliva (around 99\%). The levels of hNP-4 are roughly 100 folds lower. The hNP1 or hNP2 actively destroy Staphylococcus aureus, Pseudomonas aeruginosa and Escherichia coli as compared to hNP3 and hNP4 which are active against Candida albicans, E. coli and Streptococcus faecalis $[5,9]$.

In the $\beta$-defensin subfamily only $\mathrm{hBD}-1,-2$ and -3 are substantially expressed in the oral cavity. HBD-1 and hBD-2 peptides are localized in differentiated epithelial cells inside the suprabasal layers of standard gingival epithelium, whereas hBD-3 peptide is expressed in undifferentiated epithelial cells within the basal layer, signifying a potential role for hBD-3 as 
a mediator to signal the underlying connective tissue cells [2]. hBD1 obstructs normal flora from becoming opportunistic and is expressed continuously; on the other hand, hBD2 and3 are more effective against almost all pathogens and are induced in response to bacterial lipopolysaccharides (LPS), tumor necrosis factors $(\mathrm{TNF} \alpha)$, proinflammatory mediators (interleukins1- $\beta$ [IL1 $\beta]$ and interferons [8].

\section{Cathelicidins (LL37)}

These belong to the $\alpha$ helical peptides family, do not have cysteine and are located at the carboxyl terminus of a 15-18kDa highly conserved cathepsinLinhibitor (cathelin)like domain [7]. It is a multifunctional peptide, comprising of 37 amino acids. Active against gram positive and gram negative bacteria's, it directly binds to the LPS of bacterial cells. By activating antigen-presenting cells, it presents as a hemoattractant for immune cells, including monocytes, $\mathrm{T}$ cells, etc. There is a specific correlation amongst multiplication in LL-37 levels and periodontal inflammation. Their mode of action involves intracellular killing of the phagosomes after phagocytosis of the bacteria, where the AMP in the neutrophil is severed into a fully developed peptide.

\section{Histatins}

Histatins are a family of salivary proteins with low molecular weight cationic peptides synthesized by the parotid and submandibular salivary ducts cells at around $50-425 \mathrm{lg} / \mathrm{ml}$ in healthy adults. Histamine 1, 3 and 5 are found to be predominant of the total histatin proteins (85\%) in the saliva. They have a major role in fungicidal activity, having a noteworthy role in oral candidiasis restraint, especially histatin-5, which also has bactericidal activities against Porphyromonas gingivalis and Streptococcus mutans $[8,10]$.

\section{Adrenomedullin}

Adrenomedullin is a cationic amphipathic peptide with one disulfide bond. This HDP is present mostly in the GCF and saliva with larger concentrations in whole saliva approximately 55-65pg/mL. It is effective against both Grampositive and Gramnegative bacteria of the oral cavity. Adrenomedullin is increased in periodontally affected sites as compared to healthy sites [10].

\section{Statherin}

Statherin is a $5.4 \mathrm{kDa}$ peptide belonging to the histatin/ statherin family present in GCF and saliva. This HDP hinders the growth of anaerobic bacteria isolated from the oral cavity and also restrains the crystallization of calcium phosphate thus preventing plaque formation.

\section{Azurocidin}

It is a $37 \mathrm{kDa}$ cationic antimicrobial protein expressed in azurophil granules of neutrophils. Azurocidin is a 251amino acid protein and consists of two cysteine residues in positions 52 and 68. They have a strong affinity for LPS and therefore exhibit strong antibacterial properties towards Gramnegative bacteria.

\section{C-C motif chemokine 28}

This is a 128-amino acid peptide, which is principally expressed in a variety of epithelial cells, and salivary glands. C-C motif chemokine acts both as a broad-spectral antimicrobial agent and as chemokine $[5,11]$.

\section{Conclusion}

HDPs are diverse structural molecules exhibiting immunomodulatory activities including modulating pro and antiinflammatory responses, enhancing chemoattraction, killing of extracellular and intracellular bacteria, promoting cellular differentiation, activating the innate and adaptive compartments, promoting wound-healing. AMPs amalgamate with other inflammatory proteins and maintain inflammatory molecules and pathways. These peptides are peculiar in keeping the level of bacteria in control, having distinctive as well as dual roles in maintaining oral health.

\section{References}

1. Krisanaprakornkit S, Khongkhunthian S (2010) The role of antimicrobial peptides in periodontal disease (Part I): An overview of human defensins and cathelicidin. Thai J Periodontol 1: 33-44.

2. Mishra A, Apeksha B, Koppolu P, Lingam SA (2015) Role of antimicrobial peptides in periodontal innate defense mechanism. Journal of Oral Research and Review 7(2): 74-76.

3. Mansour SC, Pena OM, Hancock RE (2014) Host defense peptides: frontline immunomodulators. Trends Immunol 35(9): 443-450.

4. Gorr SU, Abdolhosseini M (2011) Antimicrobial peptides and periodontal disease. Journal of Clinical Periodontology 38 Suppl 11: 126-141.

5. Khurshid Z, Naseem M, Sheikh Z, Najeeb S, Shahab S, et al. (2016) Oral antimicrobial peptides: Types and role in the oral cavity. Saudi Pharm J 24(5): 515-524

6. Jönsson D (2018) Antimicrobial peptides: Roles in periodontal health and disease. Pathogenesis of Periodontal Diseases, pp: 97-110.

7. Ved V, Fernandes G (2017) Anti-microbial peptides and their speculative role in periodontitis. Biomedical Journal of Scientific \& Technical Research1(6): 1802-1807.

8. Gupta S, Bhatia G, Sharma A, Saxena S (2018) Host defense peptides: An insight into the antimicrobial world. J Oral Maxillofac Pathol 22(2): 239-244.

9. Bulet P, Stöcklin R, Menin L (2004) Anti-microbial peptides: from invertebrates to vertebrates. Immunol Rev 198: 169-184.

10. Mallapragada S, Wadhwa A, Agrawal P (2017) Antimicrobial peptides: The miraculous biological molecules. J Indian Soc Periodontol 21(6): 434-438.

11. Wiesner J, Vilcinskas A (2010) Antimicrobial peptides: the ancient arm of the human immune system. Virulence 1(5): 440-464. 